\title{
More about spaces with a small diagonal
}

\author{
by
}

Alan Dow and Oleg Pavlov (Charlotte, NC)

\begin{abstract}
Hušek defines a space $X$ to have a small diagonal if each uncountable subset of $X^{2}$ disjoint from the diagonal has an uncountable subset whose closure is disjoint from the diagonal. Hušek proved that a compact space of weight $\omega_{1}$ which has a small diagonal will be metrizable, but it remains an open problem to determine if the weight restriction is necessary. It has been shown to be consistent that each compact space with a small diagonal is metrizable; in particular, Juhász and Szentmiklóssy proved that this holds in models of $\mathrm{CH}$. In the present paper we prove that this also follows from the Proper Forcing Axiom (PFA). We furthermore present two (consistent) examples of countably compact non-metrizable spaces with small diagonal, one of which maps perfectly onto $\omega_{1}$.
\end{abstract}

1. Introduction. We refer the reader to Gruenhage's interesting article [Gru02] for more background on spaces with small diagonal. In particular, as mentioned there, H. X. Zhou [Zho82] is responsible for broadening the question to countably compact and Lindelöf spaces, while Hušek originally asked about compact and $\omega_{1}$-compact spaces. It has already been shown to hold in some models that compact spaces with small diagonal are metrizable (see [Zho82, Dow88a, Dow89, JS92]). As mentioned in the abstract, we prove that PFA implies that compact spaces with small diagonal are metrizable. This is the content of the second section. In the third section, we present two constructions of countably compact spaces with small diagonals. The first, from the hypothesis $\diamond^{+}$, maps perfectly onto $\omega_{1}$ with metric fibers. The second example is presented because the set-theoretic hypothesis that we are able to use is quite weak.

In this section we review some of the already established results concerning spaces with small diagonal that will be useful in our proofs. It is easily seen that a space with a $G_{\delta}$-diagonal has a small diagonal. The Sorgenfrey line is a well known example of a Lindelöf space with a $G_{\delta^{-}}$-diagonal which

2000 Mathematics Subject Classification: Primary 54D20.

Key words and phrases: PFA, countably compact, small diagonal, metrizable.

The first author acknowledges support provided by NSF grant DMS-0103985. 
is not metrizable. However for countably compact spaces the following very interesting result is well known.

Proposition 1 ([Cha76]). A countably compact space with a $G_{\delta}$-diagonal is metrizable.

Proposition 2 ([JS92]). A compact space with a small diagonal has countable tightness.

Proposition 3 ([Dow88b]). A countably compact space is metrizable if each of its subspaces of cardinality at most $\aleph_{1}$ is metrizable.

Proposition 4 ([Huš77]). A compact space of weight at most $\aleph_{1}$ is metrizable if it has a small diagonal.

Corollary 5. If a compact space has a small diagonal, then it is metrizable if each of its separable subspaces is metrizable.

Proof. By Proposition 3, we may assume that $X$ has a dense subset of cardinality at most $\aleph_{1}$ and, by Proposition 2, that $X$ has countable tightness. Therefore $X$ can be written as an increasing union of compact separable subspaces. If each of these is metrizable, then each has countable weight. In addition, $X$ would then have a net of cardinality $\aleph_{1}$. Since the weight of a compact space is equal to the minimum cardinality of a net (i.e. weight is equal to net weight), $X$ would have weight at most $\omega_{1}$, and so by Proposition 4, $X$ would be metrizable.

Proposition 6 ([Gru02]). A first-countable hereditarily Lindelöf space $X$ with a small diagonal will have a $G_{\delta}$-diagonal.

2. PFA and compact spaces with small diagonal. As is well known, it follows from PFA that there is no S-space.

Proposition 7 ([Tod89]). PFA implies that each hereditarily separable $(h S)$ space is also hereditarily Lindelöf $(h L)$.

Proposition 8 ([Tod89]). PFA implies OCA: if $X$ is a separable metric space and $K_{0}$ is a symmetric open subset of $X^{2} \backslash \Delta_{X}$, then either there is an uncountable $Y \subset X$ such that $Y^{2} \backslash \Delta_{X} \subset K_{0}$, or $X$ can be covered by $a$ countable family $\left\{X_{n}: n \in \omega\right\}$ such that for each $n, X_{n}^{2}$ is disjoint from $K_{0}$.

Our main result of this section is the following theorem.

THEOREM 9. PFA implies that each compact space with a small diagonal is metrizable.

By Corollary 5, we may assume that our space of interest is separable. We will use the following characterization from [Gru02, 1.2].

Proposition 10. A Lindelöf space $X$ has a small diagonal iff for each uncountable family $\left\{\left(x_{\alpha}, y_{\alpha}\right): \alpha \in \omega_{1}\right\}$ of pairs of distinct points of $X$ there 
is an uncountable $A \subset \omega_{1}$ such that $\left\{x_{\alpha}: \alpha \in A\right\}$ and $\left\{y_{\alpha}: \alpha \in A\right\}$ are separated by disjoint open $F_{\sigma}$-sets.

The following result is established by proving that $f$ is a closed mapping on $Y$, hence a homeomorphism.

Proposition 11. If $X$ is countably compact and $f$ is a continuous function from $X$ into a metric space and $Y \subset X$ is such that $f$ is one-to-one on $Y$ (i.e. $f^{-1}(f(y))=\{y\}$ for each $\left.y \in Y\right)$, then $Y$ is metrizable.

In case it may have independent interest, we prove the following strengthening of Theorem 9. The reader will recall that Balogh has shown that PFA implies that each compact space of countable tightness is sequential.

LEMMA 12. PFA implies that each separable non-metrizable sequentially compact space $X$ will contain a family of pairs $\left\{\left(y_{\alpha}, z_{\alpha}\right): \alpha \in \omega_{1}\right\}$ such that for each uncountable $A \subset \omega_{1},\left\{y_{\alpha}: \alpha \in A\right\}$ and $\left\{z_{\alpha}: \alpha \in A\right\}$ are not separated by disjoint open sets.

Proof. We may assume that $\omega$ is a dense subset of our space $X$ and that $X$ is embedded in $[0,1]^{\kappa}$ for some cardinal $\kappa$. We know by Proposition 3 that $X$ must contain a non-metrizable subspace of cardinality $\omega_{1}$, but we will choose a special subspace.

If $X$ were hL, then it would be compact and first countable, hence by Proposition $6, X$ would be metrizable. Therefore $X$ is not $\mathrm{hL}$, so it has a right-separated subspace $\left\{x_{\alpha}: \alpha \in \omega_{1}\right\}$ (i.e. for each $\alpha<\omega_{1}$, the set $\left\{x_{\beta}: \beta<\alpha\right\}$ is relatively open). Additionally, since we are assuming PFA, by Proposition 7 the subspace $\left\{x_{\alpha}: \alpha<\omega_{1}\right\}$ cannot be hS, hence it has an uncountable discrete subspace. Therefore we may assume that $\left\{x_{\alpha}: \alpha \in \omega_{1}\right\}$ is a discrete subspace of $X$. For each $\alpha<\omega_{1}$, let $W_{\alpha}, U_{\alpha}$ be open subsets of $X$ so that $x_{\alpha} \in W_{\alpha} \subset \bar{W}_{\alpha} \subset U_{\alpha}$, and $\bar{U}_{\alpha} \cap\left\{x_{\beta}: \alpha \neq \beta<\omega_{1}\right\}$ is empty.

For each $\alpha \neq \gamma, x_{\alpha} \notin \bar{U}_{\gamma}$, hence the family $\left\{\omega \cap W_{\alpha} \backslash \bar{U}_{\gamma}: \gamma \neq \alpha\right\}$ has the finite intersection property. Since PFA implies MA $\left(\omega_{1}\right)$, there is an infinite set $a_{\alpha} \subset W_{\alpha} \cap \omega$ such that $a_{\alpha} \cap \bar{U}_{\gamma}$ is finite for all $\gamma \neq \alpha$. Since $X$ is sequentially compact, we may assume that there is a $y_{\alpha} \in \bar{W}_{\alpha}$ such that $a_{\alpha}$ converges to $y_{\alpha}$. Furthermore, by enlarging $W_{\alpha}$ by a small amount, we may assume that $y_{\alpha} \in W_{\alpha} \subset \bar{W}_{\alpha} \subset U_{\alpha}$.

Now we have chosen an uncountable discrete set $Y=\left\{y_{\alpha}: \alpha \in \omega_{1}\right\}$ and a family of sequences $a_{\alpha} \subset \omega$ such that $a_{\alpha}$ converges to $y_{\alpha}$. It of course follows that $\omega \cup Y$ is not metrizable. We are ready to apply OCA. Let $\mathcal{X}$ denote the family of all pairs $(a, b)$ of subsets of $\omega$ such that there is an $\alpha \in \omega_{1}$ and a $z \in X \backslash Y$ such that $a=a_{\alpha}, a \cap b$ is empty, and $b$ converges to $z$.

We topologize $\mathcal{X}$ by the usual separable metric topology where for each $n \in \omega,\left[(a, b)_{n}\right]$ denotes the family of all pairs $\left(a^{\prime}, b^{\prime}\right) \in \mathcal{X}$ such that $a^{\prime} \cap n=$ $a \cap n$ and $b^{\prime} \cap n=b \cap n$. 
Define a set $K_{0} \subset \mathcal{X}^{2}$ by $\left(\left(a_{\alpha}, b_{\alpha}\right),\left(a_{\beta}, b_{\beta}\right)\right) \in K_{0}$ if $\left(a_{\alpha} \cap b_{\beta}\right) \cup\left(a_{\beta} \cap b_{\alpha}\right)$ is not empty. Notice that it follows that $\alpha \neq \beta$ since otherwise $\left(a_{\alpha} \cup a_{\beta}\right) \cap$ $\left(b_{\alpha} \cup b_{\beta}\right)$ would be empty.

One can see that $K_{0}$ is open by simply choosing $n$ large enough so that $\left(a_{\alpha} \cap b_{\beta}\right) \cup\left(a_{\beta} \cap b_{\alpha}\right)$ has a point in it below $n$.

Assume that $A \subset \omega_{1}$ is uncountable and $\left\{\left(a_{\alpha}, b_{\alpha}\right): \alpha \in A\right\}$ is such that

$$
\left(\left(a_{\alpha}, b_{\alpha}\right),\left(a_{\beta}, b_{\beta}\right)\right) \in K_{0} \quad \text { for each } \alpha \neq \beta \in A .
$$

For each $\alpha \in A$, let $z_{\alpha} \in X$ be the point such that $b_{\alpha}$ converges to $z_{\alpha}$.

Claim 1. The family $\left\{\left(y_{\alpha}, z_{\alpha}\right): \alpha \in A\right\}$ satisfies the conclusion of the lemma.

Assume that $U, V$ are disjoint open subsets of $X$ such that $y_{\alpha} \in U$ and $z_{\alpha} \in V$ for all $\alpha \in A^{\prime}$ for some uncountable $A^{\prime} \subset A$. By shrinking $A^{\prime}$ we may assume that there is an $n \in \omega$ such that $a_{\alpha} \backslash U \subset n$ and $b_{\alpha} \backslash V \subset n$ for all $\alpha \in A^{\prime}$. Furthermore, we may assume that $a_{\alpha} \cap n$ and $b_{\alpha} \cap n$ are independent of $\alpha \in A^{\prime}$. Clearly then, if $\alpha, \beta$ are distinct members of $A^{\prime}$ we see that $n \cap a_{\alpha} \cap b_{\beta}=n \cap a_{\beta} \cap b_{\beta}$ is empty, and $a_{\alpha} \backslash n \subset U$ while $b_{\beta} \backslash n \subset V$, contradicting that $\left(\left(a_{\alpha}, b_{\alpha}\right),\left(a_{\beta}, b_{\beta}\right)\right) \in K_{0}$.

To finish the proof, we assume that $\mathcal{X}_{n}$ are subsets of $\mathcal{X}$ with the property that $\mathcal{X}_{n}^{2}$ is disjoint from $K_{0}$ and show that $\mathcal{X}$ is not equal to $\bigcup_{n} \mathcal{X}_{n}$. For each $n$, let $Y_{n}=\bigcup\left\{a_{\alpha}:(\exists b)\left(a_{\alpha}, b\right) \in \mathcal{X}_{n}\right\}$.

Let $M$ be a countable elementary submodel of $H(\theta)$ for some large enough regular cardinal $\theta$ and assume that $\left\{y_{\alpha}: \alpha \in \omega_{1}\right\},\left\{a_{\alpha}: \alpha \in \omega_{1}\right\}$, $\left\{W_{\alpha}: \alpha \in \omega_{1}\right\},\left\{\mathcal{X}_{n}: n \in \omega\right\}$, and the embedding of $X$ as a subspace of $[0,1]^{\kappa}$ are each elements of $M$.

Let $f$ denote the projection map from $X$ into $[0,1]^{M \cap \kappa}$. Let $\delta=M \cap \omega_{1}$ (the minimum ordinal not in $M$ ). Let $\left\{U_{m}: m \in \omega\right\}$ be a descending family of basic open subsets of $[0,1]^{\kappa \cap M}$ (using rational intervals in finitely many cooordinates) which form a neighborhood base at $f\left(y_{\delta}\right)$. Although the family $\left\{U_{m}: m \in \omega\right\}$ is actually disjoint from $M$, the set $f^{-1}\left(U_{m}\right)$, for each $m$, is an open subset of $X$ which is an element of $M$. Note that for $\beta \in M \cap \omega_{1}$, $f\left(y_{\beta}\right) \neq f\left(y_{\delta}\right)$ since $W_{\beta} \in M$ and $f\left(y_{\gamma}\right) \notin f\left(W_{\beta}\right)$ for all $\gamma>\beta$.

Let $J=\left\{n \in \omega: a_{\delta} \subset Y_{n}\right\}$.

Claim 2. There is an infinite set $b \subset \omega$ converging to a point $z^{\prime} \in X \backslash Y$ such that for each $m \in \omega$,

$$
b \backslash \bigcap_{n \in J \cap m} Y_{n} \text { is finite. }
$$

To find our set $b$ we again use $\operatorname{MA}\left(\omega_{1}\right)$ to assert that there is a subset $b \subset \omega \backslash a_{\delta}$ such that $b$ is almost contained in $\bigcap_{n \in J \cap m} Y_{n} \cap f^{-1}\left(U_{m}\right) \backslash\left(W_{\delta} \cup W_{\gamma}\right)$ for each $\gamma>\delta$ and $m \in \omega$. 
To see this it is sufficient to show that

$$
\bigcap_{n \in J \cap m} Y_{n} \cap f^{-1}\left(U_{m}\right) \backslash\left(\bar{W}_{\gamma_{0}} \cup \cdots \cup \bar{W}_{\gamma_{l}}\right)
$$

is infinite for each $l \in \omega$ and $\delta \leq \gamma_{0}<\cdots<\gamma_{l}<\omega_{1}$.

This again follows from the fact that $a_{\delta}$ is almost contained in the set $A=\bigcap_{n \in J \cap m} Y_{n} \cap f^{-1}\left(U_{m}\right)$, which is in $M$. By elementarity, there is a $\beta \in M$ such that $a_{\beta}$ is almost contained in $A$. Since such an $a_{\beta}$ is almost disjoint from each $W_{\gamma}$ with $\gamma \geq \delta$, we have shown that the above set is infinite.

We may shrink $b$ so that it converges to some $z \in X$ and we observe that $f(z)=f\left(y_{\delta}\right)$. We check that $z \notin Y$. Since $b$ is almost disjoint from $W_{\gamma}$ for all $\gamma \geq \delta$, we have $z \neq y_{\gamma}$ for all $\gamma \geq \delta$. On the other hand, our construction has ensured that $f\left(y_{\beta}\right) \neq f\left(y_{\delta}\right)$ for each $\beta \in M$.

Finally then, we have produced a pair $\left(a_{\delta}, b\right) \in \mathcal{X}$. Assume that $\left(a_{\delta}, b\right)$ $\in \mathcal{X}_{n}$, hence $a_{\delta} \subset Y_{n}$. By construction, it follows that $n \in J$, hence $b$ is almost contained in $Y_{n}$. Choose any $j \in b \cap Y_{n}$ and fix $\left(a_{\beta}, b_{\beta}\right) \in \mathcal{X}_{n}$ such that $j \in a_{\beta}$. Since $b \cap a_{\delta}$ is empty, it follows that $\beta \neq \delta$. However, we see that $\left(a_{\beta} \cap b\right) \cup\left(a_{\delta} \cap b_{\beta}\right)$ is not empty, which means that the pair $\left(\left(a_{\delta}, b\right),\left(a_{\beta}, b_{\beta}\right)\right)$ is in $K_{0}$, a contradiction.

3. Countably compact with small diagonal. Gruenhage has shown that it is consistent with $\mathrm{CH}$ that there is no countably compact nonmetrizable space with a small diagonal [Gru02, 4.2]. He has also shown that it is consistent with the failure of $\mathrm{CH}$ that there is a countably compact non-metrizable space with a small diagonal [Gru02, 4.3]. In this section we generalize these results in two ways. In the first result we prove that it is consistent with $\mathrm{CH}$ that there is a perfect first-countable preimage of $\omega_{1}$ (with metrizable fibers) which has a small diagonal (Theorem 13). This example answers several of the questions in [Gru02]. Secondly, we establish, from a much weaker set-theoretic hypothesis than that in [Gru02, 4.3], that there is a countably compact non-metrizable space with a small diagonal (Theorem 16).

Recall that the classical $\diamond^{+}$(see $[\mathrm{Ku} 80]$ ) is the following statement:

There are sets $\mathcal{A}_{\alpha} \subset \mathcal{P}(\alpha)$, for $\alpha<\omega_{1}$, such that each $\left|\mathcal{A}_{\alpha}\right| \leq \omega$ and, for each $A \subset \omega_{1}$, there is a club $F \subset \omega_{1}$ such that for every $\alpha \in F, A \cap \alpha \in \mathcal{A}_{\alpha}$ and $F \cap \alpha \in \mathcal{A}_{\alpha}$.

THEOREM $13\left(\diamond^{+}\right)$. There is a perfect preimage of $\omega_{1}$ with a small diagonal.

Proof. Let $C=\{0,1\}^{\omega}$ be a copy of a Cantor set. Let $X=\omega_{1} \times C$. Let $\pi_{1}$ and $\pi_{2}$ denote projections of $X$ onto $\omega_{1}$ and $C$ respectively. For every $\alpha \in \omega_{1}, C_{\alpha}, X_{<\alpha}$, and $X_{\leq \alpha}$ are subsets of $X$, where $C_{\alpha}=\{\alpha\} \times C$, 
$X_{<\alpha}=\alpha \times C$, and $X_{\leq \alpha}=(\alpha+1) \times C$. We put $X_{<\omega_{1}}=X$. Let $\phi_{1}$ and $\phi_{2}$ be the projections of $X^{2}$ onto the corresponding coordinates.

We define a topology on $X$ in such a way that $\pi_{1}$ is a continuous perfect map. We define the topology at points of $C_{\alpha}$ recursively for $\alpha \in \omega_{1}$. Each $X_{\leq \alpha}$ will be a subspace and an open subset of $X$, so a typical neighborhood of $x \in C_{\alpha}$ in $X_{\leq \alpha}$ will be a typical neighborhood of this $x$ in $X$.

We introduce some notation for the properties that will ensure that $X$ will have a small diagonal. A set $A \subset X^{2}$ will be said to be simple if $\pi_{1}\left(\phi_{1}(A)\right) \cup \pi_{1}\left(\phi_{2}(A)\right)$ is discrete and for $a \neq a^{\prime}$ both in $A$, either $\max \left\{\pi_{1}\left(\phi_{2}(a)\right), \pi_{1}\left(\phi_{2}(a)\right)\right\}<\min \left\{\pi_{1}\left(\phi_{2}\left(a^{\prime}\right)\right), \pi_{1}\left(\phi_{2}\left(a^{\prime}\right)\right)\right\}$ or vice versa. A simple set $A$ is well-ordered by the map sending $a \in A$ to $\min \left\{\pi_{1}\left(\phi_{2}(a)\right)\right.$, $\left.\pi_{1}\left(\phi_{2}(a)\right)\right\}$.

We can reformulate $\diamond^{+}$to a more suitable form as follows. Let $h$ be any function from $\omega_{1}$ onto $\omega_{1} \cup X^{2}$ such that for each limit ordinal $\delta, h(\delta)=\delta$ and for each $\alpha<\omega_{1}, h(\{\beta: \beta<\alpha\}) \subset \alpha \cup X_{<\alpha}^{2}$. Let $\left\{\mathcal{A}_{\alpha}: \alpha \in \omega_{1}\right\}$ be the $\diamond^{+}$-sequence as above. For each $\alpha$, let $\mathcal{A}_{\alpha}^{\prime}$ be those $A \in \mathcal{A}_{\alpha}$ such that $h(A)$ is a simple set (contained in $\left.\left(X_{<\alpha}\right)^{2}\right)$. Also, let $\mathcal{B}_{\alpha}$ be those $B \in \mathcal{A}_{\alpha}$ such that $B$ is a closed set of limit ordinals. With no loss of generality, we may assume that each of $\mathcal{A}_{\alpha}^{\prime}$ and $\mathcal{B}_{\alpha}$ is infinite for each infinite ordinal $\alpha$.

Now, for each infnite $\alpha \in \omega_{1}$, let $\left\{B_{\alpha}^{n}: n \in \omega\right\}$ enumerate the set $\mathcal{B}_{\alpha}$, and let $\left\{A_{\alpha}^{n}: n \in \omega\right\}$ enumerate the set $\left\{h(A): A \in \mathcal{A}_{\alpha}^{\prime}\right\}$. It is a routine exercise to verify that the following will hold:

There are sequences $\mathcal{A}=\left\{A_{\alpha}^{m} \subset\left(X_{<\alpha}\right)^{2}: \alpha \in \omega_{1}, m \in \omega\right\}$ and $\mathcal{B}=$ $\left\{B_{\alpha}^{n} \subset \alpha: \alpha \in \omega_{1}, n \in \omega\right\}$ such that for every simple $A \subset X^{2}$ there are a club $F \subset \omega_{1}$ and sequences $\left\{m(\alpha) \in \omega: \alpha \in \omega_{1}\right\}$ and $\left\{n(\alpha) \in \omega: \alpha \in \omega_{1}\right\}$ such that for every $\alpha \in F, A \cap\left(X_{<\alpha}\right)^{2}=A_{\alpha}^{m(\alpha)}$ and $F \cap \alpha=B_{\alpha}^{n(\alpha)}$.

The topology on $X$ will satisfy the following conditions for every $\alpha \in \omega_{1}$ :

$\left(1_{\alpha}\right) C_{\alpha}$ is homeomorphic to a Cantor set.

$\left(2_{\alpha}\right) X_{<\alpha}$ is 0 -dimensional.

$\left(3_{\alpha}\right) X_{\leq \alpha}$ is a compact space.

(4 $\left.4_{\alpha}\right) \pi_{1\left\lceil X_{<\alpha}\right.}$ is a continuous map.

$\left(5_{\alpha}\right)$ If $\alpha$ is a successor ordinal, then $C_{\alpha}$ is clopen in $X_{\leq \alpha}$.

Let $A$ be a simple subset of $X^{2}$. We say that $A$ is thin if $\overline{\phi_{1}(A)} \cap \overline{\phi_{2}(A)}=\emptyset$. For every $\beta \in \omega_{1}$ and simple $A$ we say that $A$ is simple in $\beta$ (respectively, $A$ is thin in $\beta$ ) if $A \cap\left(X_{<\beta}\right)^{2}$ is simple (respectively, thin) in the space $X_{<\beta}$. We denote by $\lim (A)$ the set $\left\{\pi_{1}\left(\phi_{1}(x)\right): x\right.$ is a limit point of $A$ in $\left.X^{2}\right\}$ $\left(=\left\{\pi_{1}\left(\phi_{2}(x)\right): x\right.\right.$ is a limit point of $A$ in $\left.\left.X^{2}\right\}\right)$. Finally, let $F \subset \gamma$ be a non-empty closed set. When the context is clear, for each $\delta \in F, \delta^{+}$will denote the next smallest element of $F \cup\{\gamma\}$, i.e. $\delta^{+}$is $\gamma$ if $\delta=\max (F)$. If $A \cap\left(X_{<\delta^{+}} \backslash X_{<\delta}\right)^{2}$ is a non-empty set, denote by $a_{\delta}$ the smallest element 
of this set (according to the induced well-ordering of $A$ as a simple set). If $A \cap\left(X_{<\delta^{+}} \backslash X_{<\delta}\right)^{2}$ is empty, we put $a_{\delta}=\emptyset$. Denote $\left\{a_{\delta}: \delta \in F\right\}$ by $A_{\uparrow F}$. If $F$ is empty, let $A_{\lceil F}$ be empty as well.

Consider the following condition on a pair $(m, n) \in \omega^{2}$ :

$\left(*_{\alpha}\right) A_{\alpha}^{m}$ is simple in $\alpha, B_{\alpha}^{n}$ is closed in $\alpha$, and $A_{\alpha \uparrow B_{\alpha}^{n} \cap \lim \left(A_{\alpha}^{m}\right)}^{m}$ is thin in $\alpha$.

The following condition will ensure that $X$ has a small diagonal:

$\left(6_{\alpha}\right)$ If $(m, n)$ satisfies $\left(*_{\alpha}\right)$, then $A_{\alpha\left\lceil B_{\alpha}^{n} \cap \lim \left(A_{\alpha}^{m}\right)\right.}^{m}$ is thin in $\alpha+1$.

Note that if $(m, n)$ satisfies $\left(*_{\gamma}\right)$, then condition $\left(6_{\gamma}\right)$ essentially means that $\gamma$ is not the minimal ordinal such that

$$
\overline{\phi_{1}\left(A _ { \gamma } ^ { m } \left\lceilB_{\gamma}^{n} \cap \lim \left(A_{\gamma}^{m}\right)\right.\right.} \cap \overline{\phi_{2}\left(A _ { \gamma } ^ { m } \left\lceilB_{\gamma}^{n} \cap \lim \left(A_{\gamma}^{m}\right)\right.\right.} \cap C_{\gamma} \neq \emptyset .
$$

Using $\left(6_{\alpha}\right)$, we will show that for every uncountable simple subset $A$ of $X^{2}, \overline{\phi_{1}\left(A_{\lceil G}\right)} \cap \overline{\phi_{2}\left(A_{\lceil G}\right)}=\emptyset$ since there is no minimal $\gamma \in \omega_{1}$ such that $\overline{\phi_{1}\left(A_{\lceil G}\right)} \cap \overline{\phi_{2}\left(A_{\uparrow G}\right)} \cap C_{\gamma} \neq \emptyset$ (here $G$ is a certain club in $\omega_{1}$ which depends on $A$ and which is produced with the help of $\diamond^{+}$-sequences).

Now let $\gamma \in \omega_{1}$ and suppose that a topology has been defined on $X_{<\gamma}$ and satisfies $\left(1_{\alpha}\right)-\left(6_{\alpha}\right)$ for every $\alpha<\gamma$.

CASE 1: $\gamma$ is a successor ordinal. In this case, let $C_{\gamma}$ be a clopen subset of $X_{\leq \gamma}$ which is homeomorphic to a Cantor set. Then $\left(1_{\gamma}\right)-\left(6_{\gamma}\right)$ are obviously satisfied.

CAsE 2: $\gamma$ is a limit ordinal. In this case, $X_{<\gamma}$ is a free topological sum of sets with zero large inductive dimension, so $\left(2_{\gamma}\right)$ holds. Enumerate all pairs $(m, n) \in \omega^{2}$ which satisfy $\left(*_{\gamma}\right)$ by $\left\{\left(m_{k}, n_{k}\right): k \in \omega\right\}$. We can assume that the family of such pairs is infinite (if not, replace some $A_{\gamma}^{m}, B_{\gamma}^{n}$ so that infinitely many pairs $(m, n) \in \omega^{2}$ satisfy $\left.\left(*_{\gamma}\right)\right)$. For every $k \in \omega$, let $\mathcal{I}_{k}=\{0,1\}^{k}$ be the set of all binary sequences of length $k$, and let $\mathcal{I}=\bigcup\left\{\mathcal{I}_{k}: k \in \omega\right\}$. Let $U_{0}, U_{1}$ be complementary clopen subsets of $X_{<\gamma}$ such that $\phi_{1}\left(A_{\gamma}^{m_{0}}\left\lceil B_{\gamma}^{n_{0}} \cap \lim \left(A_{\gamma}^{m_{0}}\right)\right.\right.$ $\subset U_{0}$ and $\phi_{2}\left(A_{\gamma}^{m_{0}}{ }_{i B_{\gamma}^{n_{0}} \cap \lim \left(A_{\gamma}^{m_{0}}\right)}\right) \subset U_{1}$. Such sets exist according to $\left(2_{\gamma}\right)$ and $\left(*_{\gamma}\right)$. Now assume that a family $\left\{U_{I}: I \in \mathcal{I}_{k}\right\}$ of clopen subsets of $X_{<\gamma}$ has been defined for some $k \in \omega$. For every $I \in \mathcal{I}_{k}$, let $U_{I^{\curlyvee} 0}, U_{I^{\wedge} 1}$ be complementary clopen subsets of $U_{I}$ such that $\phi_{1}\left(A_{\gamma}^{m_{k}}\left\lceil B_{\gamma}^{n_{k}} \cap \lim \left(A_{\gamma}^{m_{k}}\right) \cap U_{I}\right.\right.$ $\subset U_{\Gamma^{\curlyvee} 0}$ and $\phi_{2}\left(A_{\gamma}^{m_{k}}{ }_{\left\lceil B_{\gamma}{ }^{n} \cap \lim \left(A_{\gamma}^{m_{k}}\right)\right.}\right) \cap U_{I} \subset U_{\Gamma^{\wedge} 1}$. Let $c=\left\{i_{p} \in\{0,1\}: p \in\right.$ $\omega\}$ be an element of $C$. Then a typical neighborhood of $(\gamma, c)$ in $X_{\leq \gamma}$ is $\left(\{\gamma\} \times C_{I}\right) \cup\left(U_{I} \backslash X_{\leq \delta}\right)$ for various $I \in \mathcal{I}$ and $\delta<\gamma$, where $C_{I}$ denotes the set of all elements of $C$ which extend $I$.

Now we check properties $\left(1_{\gamma}\right)$ and $\left(3_{\gamma}\right)-\left(6_{\gamma}\right)$ in Case 2. 
It is well known that $\left\{C_{I}: I \in \mathcal{I}\right\}$ is a base of $C$. Hence $\left\{\{\gamma\} \times C_{I}\right.$ : $I \in \mathcal{I}\}$ is a base of $C_{\gamma}$. Therefore $C_{\gamma}$ is homeomorphic to a Cantor set and $\left(1_{\gamma}\right)$ holds.

Assume towards a contradiction that $\left(3_{\gamma}\right)$ fails. Since $X_{\leq \gamma}$ is separable metrizable, there is a countably infinite subset $S$ of $X_{\leq \gamma}$ without an accumulation point in $X_{\leq \gamma}$. Therefore for every $x \in C_{\gamma}$, there is $I(x) \in \mathcal{I}$ such that the sets $U_{I(x)} \cap S$ and $C_{I(x)} \cap S$ are finite. The family $\left\{C_{I(x)}: x \in C_{\gamma}\right\}$ is an open cover of $C_{\gamma}$, so it contains a finite subcover, which we denote by $\mathcal{U}$. It then follows from the definition of topology in $C_{\gamma}$ that $\cup \mathcal{U}$ contains $X_{\leq \gamma} \backslash X_{\leq \delta}$ for some $\delta<\gamma$. Therefore $S \cap X_{\leq \delta}$ is an infinite subset of $X_{\leq \delta}$ with accumulation point in $X_{\leq \delta}$. However, $X_{\leq \delta}$ is a compact space according to $\left(3_{\delta}\right)$. This contradiction proves $\left(3_{\gamma}\right)$.

$\left(4_{\gamma}\right)$ is true since $\left(4_{\alpha}\right)$ is true for every $\alpha<\gamma$ and since $\left(\pi_{1}\right)^{-1}((\alpha, \gamma])$ is open in $X_{\leq \gamma}$ from the definition of topology on $X_{\leq \gamma}$.

$\left(5_{\gamma}\right)$ holds trivially in Case 2.

$\left(6_{\gamma}\right)$ holds because $\bigcup\left\{U_{\Gamma^{\circ} 0}: I \in \mathcal{I}_{k}\right\}$ contains $\phi_{1}\left(A_{\gamma}^{m_{k}}\left\lceil B_{\gamma}^{n_{k}} \cap \lim \left(A_{\gamma}^{m_{k}}\right)\right)\right.$ and, similarly, $\bigcup\left\{U_{I^{\curlyvee} 1}: I \in \mathcal{I}_{k}\right\}$ contains $\phi_{2}\left(A_{\gamma}^{m_{k}}{ }_{i B_{\gamma}^{n_{k}} \cap \lim \left(A_{\gamma}^{m_{k}}\right)}\right)$.

We next show that $\pi_{1}$ is a continuous perfect map. Fix $x \in X$. Then there is $\alpha \in \omega_{1}$ such that $x \in X_{<\alpha}$. But $X_{<\alpha}$ is an open neighborhood of $x$ and $\pi_{1 \uparrow X_{<\alpha}}$ is a continuous map by $\left(4_{\alpha}\right)$. Therefore $\pi_{1}$ is continuous at $x$. Hence $\pi_{1}$ is a continuous map. Now fix a closed set $F \subset X$. To prove that $\pi_{1}(F)$ is closed in $\omega_{1}$, it is enough to show that $\pi_{1}(F) \cap[0, \alpha]$ is closed for every $\alpha \in \omega_{1}$. The latter statement is true since $\pi_{1}(F) \cap[0, \alpha]=\pi_{1}\left(F \cap X_{\leq \alpha}\right)$ and since $F \cap X_{\leq \alpha}$ is a compact set by $\left(3_{\alpha}\right)$. Further, every fiber of $\pi_{1}$ is a compact set by $\left(1_{\alpha}\right)$. So $\pi_{1}$ is a continuous perfect map.

The last step is to show that $X$ has a small diagonal. Recall that $\Delta(X)$ denotes the diagonal of $X$. Fix an uncountable set $A \subset X^{2}$. If $A \cap\left(X \backslash X_{\leq \delta}\right)^{2}$ is countable for some $\delta \in \omega_{1}$, then either $A \cap\left(X_{\leq \delta} \times\left(X \backslash X_{\leq \delta}\right)\right)$ is uncountable, or $A \cap\left(\left(X \backslash X_{\leq \delta}\right) \times X_{\leq \delta}\right)$ is uncountable, or $A \cap\left(X_{\leq \delta}\right)^{2}$ is uncountable. In the first two cases, $A \backslash\left(\left(X_{\leq \delta}\right)^{2} \cup\left(X \backslash X_{\leq \delta}\right)^{2}\right)$ is uncountable. In the third case, there is a neighborhood $U \subset\left(X_{\leq \delta}\right)^{2}$ of $\Delta\left(X_{\leq \delta}\right)$ such that $\left(A \cap\left(X_{\leq \delta}\right)^{2}\right) \backslash U$ is uncountable. Either way, $U \cup\left(X \backslash X_{\leq \delta}\right)^{2}$ is a neighborhood of $\Delta(X)$ which misses uncountably many elements of $A$. So we can assume that $A \cap$ $\left(X \backslash X_{\leq \delta}\right)^{2}$ is an uncountable set for every $\delta \in \omega_{1}$. Then $A$ contains an uncountable simple set. Therefore, we can just assume that $A$ is simple itself.

According to our version of $\diamond^{+}$, there is a club $F \subset \omega_{1}$ and sequences $\left\{m(\alpha) \in \omega: \alpha \in \omega_{1}\right\},\left\{n(\alpha) \in \omega: \alpha \in \omega_{1}\right\}$ such that $A \cap\left(X_{<\alpha}\right)^{2}=A_{\alpha}^{m(\alpha)}$ and $F \cap \alpha=B_{\alpha}^{n(\alpha)}$ for every $\alpha \in F$. The set $G=F \cap \lim (A)$ is a club since $\lim (A)$ is a club. We show that $A_{\lceil G}$ is an uncountable thin set. In particular, 
$\overline{A_{\lceil G}} \cap \Delta(X)=\emptyset$, which implies that $X$ has a small diagonal. First of all, for every $\delta_{1}, \delta_{2} \in G$ with $\delta_{1}<\delta_{2},\left(X_{<\delta_{2}} \backslash X_{<\delta_{1}}\right)^{2} \cap A$ is a non-empty set since $A$ is simple and since $\delta_{1}, \delta_{2} \in \lim (A)$. Therefore $A_{\uparrow G}$ is uncountable. Assume towards a contradiction that $A_{\uparrow G}$ is not thin. Then there is a minimal ordinal $\gamma \in \omega_{1}$ such that

$$
\overline{\phi_{1}\left(A_{\lceil G)}\right)} \cap \overline{\phi_{2}\left(A_{\lceil G}\right)} \cap C_{\gamma} \neq \emptyset .
$$

It is possible to check that $\gamma \in G$, in particular $\gamma \in F$. Therefore $A_{\gamma}^{m(\gamma)}=$ $A \cap\left(X_{<\gamma}\right)^{2}$ and $B_{\gamma}^{n(\gamma)} \cap \gamma=F \cap \gamma$. Also $\lim \left(A \cap\left(X_{<\gamma}\right)^{2}\right)=\lim (A) \cap \gamma$. This means that

$$
\begin{aligned}
A_{\gamma}^{m(\gamma)}{ }_{\left\lceil B_{\gamma}^{n(\gamma)} \cap \lim \left(A_{\gamma}^{m(\gamma)}\right)\right.} & =\left(A \cap\left(X_{<\gamma}\right)^{2}\right)_{\lceil(F \cap \gamma) \cap(\lim (A) \cap \gamma)} \\
& =\left(A \cap\left(X_{<\gamma}\right)^{2}\right)_{\lceil F \cap \lim (A) \cap \gamma}=\left(A \cap\left(X_{<\gamma}\right)^{2}\right)_{\lceil G \cap \gamma} \\
& =A_{\lceil G} \cap\left(X_{<\gamma}\right)^{2} .
\end{aligned}
$$

The latter set is thin in $\gamma$ by the minimality of $\gamma$. So the pair $(m(\gamma), n(\gamma))$ satisfies $\left(*_{\gamma}\right)$ and $A_{\gamma}^{m(\gamma)}\left\lceil B_{\gamma}^{n(\gamma)} \cap \lim \left(A_{\gamma}^{m(\gamma)}\right)\right)$ is thin in $\gamma+1$ by $\left(6_{\gamma}\right)$, hence

$$
\overline{\phi_{1}\left(A_{\uparrow G}\right)} \cap\left(X_{<\gamma}\right)^{2} \cap \overline{\phi_{2}\left(A_{\uparrow G}\right)} \cap\left(X_{<\gamma}\right)^{2} \cap C_{\gamma}=\emptyset .
$$

However, (2) contradicts (1) since $\phi_{i}\left(A_{\uparrow G}\right) \cap C_{\gamma}=\emptyset$ for $i \in\{1,2\}$. Therefore $A_{\lceil G}$ is thin.

For our next result, we need another set-theoretic principle.

Definition 14. The statement MA(Cohen) is the Martin's Axiom type assertion that if $P$ is a poset for adding any number of Cohen reals, i.e. for some set $I, P$ is the poset $\operatorname{Fn}(I, 2)$ of finite functions from $I$ into 2 , and if $\mathcal{D}$ is a family of fewer than $\mathfrak{c}$ dense subsets of $P$, then there is a filter $G \subset P$ which meets each member of $\mathcal{D}$.

The actual consequence of MA(Cohen) that we need is the following.

Proposition 15. (MA(Cohen)) If $\mathcal{A}$ is a family of fewer than $\mathfrak{c}$ countably infinite sets, then there is a set $Y \subset \cup \mathcal{A}$ such that neither $Y$ nor $\cup \mathcal{A} \backslash Y$ contains any of the members of $\mathcal{A}$.

The proof is of course very straightforward. One simply uses the poset $\operatorname{Fn}(\bigcup \mathcal{A}, 2)$ and with $G$ meeting $|\mathcal{A}|$ dense sets, $Y=(\bigcup G)^{-1}(0)$ works.

Theorem 16. (MA(Cohen) $\left.+2^{\omega_{1}}=2^{\omega}\right)$ Each of the spaces $2^{\mathfrak{c}}$ and $\beta \omega$ contains dense countably compact subsets of cardinality $\mathfrak{c}$ which have small diagonals.

Proof. We will define a subspace $X=\left\{x_{\alpha}: \alpha \in \mathfrak{c}\right\}$ of $2^{\mathfrak{c}}$ by induction on $\alpha \in \mathfrak{c}$. At stage $\alpha$, we will have chosen, for each $\beta<\alpha$, the values of $x_{\beta}\lceil\alpha$ for each $\beta<\alpha$. At stage $\alpha$ we will extend each $x_{\beta} \mid \alpha$ by defining its value 
at $\alpha$, i.e. $x_{\beta}(\alpha)$ will be defined to be either 0 or 1 , and we will also choose $x_{\alpha}\left\lceil\alpha+1\right.$ to be some member of $2^{\alpha+1}$.

In order to ensure that $X$ will be countably compact, let $\left\{A_{\gamma}: \gamma \in \mathfrak{c}\right\}$ be an enumeration of the countably infinite subsets of $\mathfrak{c}$ such that $A_{n}=\omega \backslash n$ for each $n \in \omega$, and $A_{\gamma} \subset \gamma$ for all $\gamma \geq \omega$. Our inductive hypothesis will include the condition, for $\gamma \leq \alpha$, that the point $x_{\gamma}\lceil\alpha$ is a limit point (in the space $2^{\alpha}$ ) of the set $\left\{x_{\beta}\left\lceil\alpha: \beta \in A_{\gamma}\right\}\right.$. We will also preserve the property that $\left\{x_{n}\lceil\alpha: n \in \omega\}\right.$ is dense in $2^{\alpha}$.

In order to ensure that $X$ will have a small diagonal, let $\left\{h_{\gamma}: \gamma \in \mathfrak{c}\right\}$ enumerate all functions from $\omega_{1}$ into $[\mathfrak{c}]^{2}$ (the two-element subsets of $\mathfrak{c}$ ) so that for each $\gamma$, the range of $h_{\gamma}$ is contained in $[\gamma]^{2}$. We will arrange that for each $\alpha$, there is an uncountable $I \subset \omega_{1}$ such that for each $\zeta \in I$, $h_{\alpha}(\zeta)=\left\{\zeta_{0}<\zeta_{1}\right\}$ and $x_{\zeta_{0}}(\alpha)=0$ and $x_{\zeta_{1}}(\alpha)=1$. Note then that if $U(\alpha, 0)$ is the subset of $2^{\mathfrak{c}}$ consisting of all points that have value 0 at $\alpha$ and $U(\alpha, 1)=2^{\mathfrak{c}} \backslash U(\alpha, 0)$, then $(U(\alpha, 0) \times U(\alpha, 0)) \cup(U(\alpha, 1) \times U(\alpha, 1))$ is an open neighborhood of the diagonal of $X$ in $X^{2}$ but contains none of the points in $\left\{\left(x_{\zeta_{0}}, x_{\zeta_{1}}\right): \zeta \in I\right.$ and $\left.h_{\alpha}(\zeta)=\left\{\zeta_{0}, \zeta_{1}\right\}\right\}$.

We begin the induction by choosing $\left\{x_{n}\lceil\omega: n \in \omega\}\right.$ to be any countable dense subset of $2^{\omega}$. At stage $\alpha \geq \omega$, we may assume that we have chosen $x_{\gamma}\left\lceil\alpha\right.$ for all $\gamma \in \alpha$ and have, by induction, preserved the property that $x_{\gamma}\lceil\alpha$ is a limit point of $\left\{x_{\beta}\left\lceil\alpha: \beta \in A_{\gamma}\right\}\right.$. We must first choose a set $J \subset \alpha$ and define $x_{\gamma}(\alpha)=0$ for all $\gamma \in J$ and $x_{\gamma}(\alpha)=1$ for all $\gamma \in \alpha \backslash J$. Then we simply choose a point $x_{\alpha}\left\lceil\alpha+1\right.$ which is a limit point of $\left\{x_{\beta}: \beta \in A_{\alpha}\right\}$.

Define $\mathcal{A}_{\alpha}$ to be the family of all countably infinite sets $B$ of $\alpha$ such that there is a basic clopen subset $W$ of $2^{\alpha}$ and a $\gamma<\alpha$ such that $B$ is a cofinite subset of $\left\{\xi \in D_{\gamma}: x_{\xi}\lceil\alpha \in W\}\right.$. Clearly $\mathcal{A}_{\alpha}$ has cardinality at most $|\alpha|$. By MA(Cohen), there is an uncountable set $I_{0}$ of $\omega_{1}$ such that $\left\{\zeta_{0}: \zeta \in I_{0}\right\}$ contains no member of $\mathcal{A}_{\alpha}$. Similarly, there is an uncountable $I_{1} \subset I_{0}$ such that $\left\{\zeta_{1}: \zeta \in I_{1}\right\}$ contains no member of $\mathcal{A}_{\alpha}$. Let $I_{2}=\left\{\zeta_{0}, \zeta_{1}: \zeta \in I_{1}\right\}$ and $\mathcal{A}_{1}$ be the family $\left\{A \backslash I_{2}: A \in \mathcal{A}_{\alpha}\right\}$ and, by MA(Cohen), choose a set $J \subset \alpha \backslash I_{2}$ so that neither $J$ nor $\alpha \backslash J$ contains any member of $\mathcal{A}_{1}$. Finally, define $x_{\gamma}(\alpha)=0$ for all $\gamma \in J \cup\left\{\zeta_{0}: \zeta \in I_{1}\right\}$, and $x_{\gamma}(\alpha)=1$ for other $\gamma \in \alpha$.

It is routine, by the choice of $\mathcal{A}_{1}$, to prove by the inductive hypothesis that $x_{\gamma}\left\lceil\alpha+1\right.$ is in the closure of $\left\{x_{\beta}\left\lceil\alpha+1: \beta \in A_{\gamma}\right\}\right.$ for each $\gamma \leq \alpha$. We have also preserved the property that $\left\{x_{n}\lceil\alpha+1: n \in \omega\}\right.$ is dense since $\mathcal{A}_{\alpha}$ will list (the indices of) all the basic clopen subsets of $2^{\alpha}$ intersected with $\left\{x_{n}\lceil\alpha: n \in \omega\}\right.$.

This completes the proof for the dense subset of $2^{\mathfrak{c}}$. One could make a minor modification of the proof in order to arrange that one particular countable discrete subset of $X$ had closure in $2^{\mathfrak{c}}$ which was homeomorphic to $\beta \omega$, and then the closure of this subset in $X$ would be our desired dense subset of $\beta \omega$. However, instead we can make one very minor additional 
requirement on our construction, namely that $x_{\gamma} \neq x_{\alpha}$ for all $\gamma \neq \alpha$. Then let $f$ be the canonical map from $\beta \omega$ onto $2^{\mathfrak{c}}$ which sends $n$ to $x_{n}$ for each $n$. For each integer $n$, let $y_{n}$ denote $n \in \beta \omega$. Recursively choose $y_{\alpha} \in \beta \omega$ (for $\alpha \in \mathfrak{c}$ ) so that $f\left(y_{\alpha}\right)=x_{\alpha}$ and $y_{\alpha}$ is a limit point of $\left\{y_{\beta}: \beta \in A_{\alpha}\right\}$. Now $f$ will be a one-to-one map from $Y=\left\{y_{\alpha}: \alpha \in \mathfrak{c}\right\}$ onto $X$ and $Y$ is easily seen to be a countably compact dense subset of $\beta \omega$. Then $Y$ has a small diagonal since the map onto $X$ is one-to-one.

It would be interesting to determine if simply the failure of $\mathrm{CH}$ implies that there is a countably compact non-metrizable space with a small diagonal. Gruenhage's example was also initially $\omega_{1}$-compact; the above construction can be modified to moreover ensure that every set of cardinality $\omega_{1}$ has a complete accumulation point by using the following stronger consequence of MA(Cohen).

Proposition 17. (MA(Cohen)) If $\mathcal{A}$ is a family of fewer than $\mathfrak{c}$ infinite sets, then there is set $Y \subset \cup \mathcal{A}$ such that for all $A \in \mathcal{A}$, each of $A \cap Y$ and $A \backslash Y$ has the same cardinality as that of $A$.

4. Lindelöf and small diagonal. In this section we present a simple observation about Lindelöf spaces with a small diagonal which generalizes a result by Zhou [Zho82] for compact spaces. We also show that the failure of $\mathrm{CH}$ implies there is a Lindelöf space with a small diagonal which does not have a $G_{\delta}$-diagonal.

Proposition 18. If a space $X$ is Lindelöf and every continuous image of $X$ has a small diagonal, then $X$ has a $G_{\delta}$-diagonal.

Proposition 18 is actually a corollary to the following.

Proposition 19. For a Lindelöf space $X$, either

(1) $X$ has a continuous image with weight (precisely) $\omega_{1}$ which does not have a small diagonal, or

(2) X can be mapped onto a metric space by a one-to-one map.

Proof. We may assume that $X$ is embedded in $[0,1]^{\kappa}$ for some cardinal $\kappa$. For each set $I \subset \kappa$, let $X_{I}$ denote the image of $X$ by the projection map $\pi_{I}:[0,1]^{\kappa} \rightarrow[0,1]^{I}$. If $I$ is countable, then of course $X_{I}$ is metrizable; hence if there is a countable $I \subset \kappa$ such that $\pi_{I} \uparrow X$ is one-to-one then the second condition holds. Therefore we assume otherwise, and inductively choose an increasing sequence $\left\{I_{\alpha}: \alpha \in \omega_{1}\right\}$ of countable sets as follows. Let $I_{0}$ be any non-empty countable subset of $\kappa$, and for limit $\alpha$ let $I_{\alpha}$ be the union $\bigcup_{\beta<\alpha} I_{\beta}$. For each $\alpha$, if $\pi_{I_{\alpha}}$ is not one-to-one, then let $x_{\alpha}, y_{\alpha}$ be a pair of points of $X$ such that $\pi_{I_{\alpha}}\left(x_{\alpha}\right)=\pi_{I_{\alpha}}\left(y_{\alpha}\right)$. Choose $I_{\alpha+1} \supset I_{\alpha}$ large enough so that $\pi_{I_{\alpha+1}}\left(x_{\alpha}\right) \neq \pi_{I_{\alpha+1}}\left(y_{\alpha}\right)$. 
Let $I=I_{\omega_{1}}$ and consider the sequence $\left\{\left(x_{\alpha}^{\prime}, y_{\alpha}^{\prime}\right): \alpha \in \omega_{1}\right\} \subset X_{I}^{2}$ where $x_{\alpha}^{\prime}=\pi_{I}\left(x_{\alpha}\right)$ and $y_{\alpha}^{\prime}=\pi_{I}\left(y_{\alpha}\right)$. To show that $X_{I}^{2}$ does not have a small diagonal, let $A$ be any uncountable subset of $\omega_{1}$. Now by the hypothesis that $X_{I}$ is Lindelöf we may choose a point $x^{\prime} \in X_{I}$ which is a complete accumulation point of the set $\left\{x_{\alpha}^{\prime}: \alpha \in A\right\}$. Of course, if $U$ is any basic open neighborhood of $x^{\prime}$ in $X_{I}$ then of the uncountably many $\alpha \in A$ with $x_{\alpha}^{\prime} \in U$, we will also have $y_{\alpha}^{\prime} \in U$ for all but countably many of them. Therefore the point $\left(x^{\prime}, x^{\prime}\right)$ on the diagonal of $X_{I}$ is a limit point of $\left\{\left(x_{\alpha}^{\prime}, y_{\alpha}^{\prime}\right)\right.$ : $\alpha \in A\}$.

Gruenhage shows in [Gru02] that it is consistent with $\mathrm{CH}$ (and holds in Gödel's constructible universe $L$ ) that there is a Lindelöf space with a small diagonal which does not have a $G_{\delta}$-diagonal. We show that the failure of $\mathrm{CH}$ alone will also provide such an example.

THEOREM 20. If $\mathfrak{c}>\omega_{1}$, then there is a Lindelöf space which has a small diagonal but not a $G_{\delta}$-diagonal.

Proof. Recall that a set $B \subset[0,1]$ is a Bernstein set if $B$ meets, but does not contain, each uncountable closed subset of $[0,1]$. It is well known that there is a family of $\mathfrak{c}$ pairwise disjoint Bernstein sets, so let $\left\{B_{\alpha}: \alpha \in \omega_{2}\right\}$ be any subfamily of such a family. Our space $X$ will simply be a subspace of the product space $\left(\omega_{2}+1\right) \times[0,1]$ where $\omega_{2}+1$ is the compact order topology on the set of ordinals. For each $\alpha<\omega_{2}$, we choose $X \cap(\{\alpha\} \times[0,1])$ to be $\{\alpha\} \times B_{\alpha}$ and we take $\left\{\omega_{2}\right\} \times[0,1] \subset X$. Basic open sets for $X$ will be taken to be of the form $X \cap([\alpha, \beta] \times I)$ for $\alpha \leq \beta \leq \omega_{2}$, with $\alpha$ not a limit ordinal, and $I$ any (relatively) open interval in $[0,1]$.

For each $\lambda \leq \omega_{2}$, we show, by induction on $\lambda$, that the subspace

$$
X_{\lambda}=X \cap([0, \lambda] \times[0,1])
$$

is Lindelöf.

If $\lambda$ has countable cofinality, then $X_{\lambda}=\bigcup_{\mu<\lambda} X_{\mu} \cup\left(\{\lambda\} \times B_{\lambda}\right)$ is a countable union of Lindelöf subsets, hence is Lindelöf itself. To see that $X_{\lambda}$ is Lindelöf when $\lambda$ has uncountable cofinality, let $\mathcal{W}$ be a cover of $X_{\lambda}$ by basic open sets. Since $\{\lambda\} \times B_{\lambda}$ (or $\left\{\omega_{2}\right\} \times[0,1]$ ) is Lindelöf, there is a countable subcollection $\mathcal{W}^{\prime}$ of $\mathcal{W}$ whose union covers $X_{\lambda} \cap(\{\lambda\} \times[0,1])$. We may assume that $\mathcal{W}^{\prime}$ is the family $\left\{\left[\alpha_{n}, \lambda\right] \times I_{n}: n \in \omega\right\}$ where each $I_{n}$ is an open subinterval of $[0,1]$. Since each $B_{\alpha}$ is Bernstein, $[0,1] \backslash \bigcup_{n} I_{n}$ is countable. In addition, the sets in the family $\left\{B_{\alpha}: \alpha<\lambda\right\}$ are pairwise disjoint (and $\lambda$ has uncountable cofinality), hence there is a $\gamma<\lambda$ such that $\alpha_{n}<\gamma$ for each $n$, and $B_{\beta} \subset \bigcup_{n} I_{n}$ for each $\gamma<\beta \leq \lambda$. It follows that $X_{\lambda} \backslash X_{\gamma}$ is contained in $\bigcup \mathcal{W}^{\prime}$. Since $X_{\gamma}$ is Lindelöf (by inductive assumption), 
it follows that $\mathcal{W}$ has a countable subcover of $X_{\lambda}$. This shows that $X_{\lambda}$ is Lindelöf.

A similar argument shows that $X$ does not have a $G_{\delta^{-}}$diagonal. Indeed, if $\left\{U_{n}: n \in \omega\right\}$ is a family of open sets in $X^{2}$ which contain $\Delta_{X}$, then for each $n$, there is a countable (finite in fact) cover, $\mathcal{W}_{n}$, of $\left\{\omega_{2}\right\} \times[0,1]$ consisting of basic open sets $W$ with the property that $W \times W \subset U_{n}$ for each $W \in \mathcal{W}_{n}$. Letting $\mathcal{W}^{\prime}$ be the collection of all the $\mathcal{W}_{n}$, choose $\gamma<\omega_{2}$ as in the above argument. Fix any $\beta$ with $\gamma<\beta<\omega_{2}$, and notice that for each $x \in B_{\beta}$, the pair $p_{x}=\left((\beta, x),\left(\omega_{2}, x\right)\right) \in X^{2}$ has the property that for each $n$, there is a $W \in \mathcal{W}_{n}$ such that $p_{x} \in W \times W \subset U_{n}$. This proves that $\Delta_{x}$ is not equal to $\bigcap_{n} U_{n}$.

Finally, we prove that $X$ has a small diagonal. Observe that the second coordinate projection map into $[0,1]$ is one-to-one on $X_{\lambda}$ for $\lambda<\omega_{2}$. Therefore, for each such $\lambda, X_{\lambda}$ is a clopen subset of $X$ which has a $G_{\delta^{-}}$diagonal. Let $A$ be an uncountable subset of $X^{2} \backslash \Delta_{X}$. If there is any $\lambda<\omega_{2}$ such that $A \cap X_{\lambda}^{2}$ is uncountable, then clearly there is an uncountable $B \subset A$ whose closure is disjoint from $\Delta_{X}$. Similarly it follows that $A$ meets the square of $\left\{\omega_{2}\right\} \times[0,1]$ in a countable set. Therefore we may assume that $A$ has cardinality $\omega_{1}$ and there is a $\lambda<\omega_{2}$ such that each point in $A$ has one point from $X_{\lambda}$ and one point from $\left\{\omega_{2}\right\} \times[0,1]$. It follows then that $X_{\lambda}^{2} \cup\left(X \backslash X_{\lambda}\right)^{2}$ is a neighborhood of $\Delta_{X}$ which is disjoint from $A$.

\section{References}

[Cha76] J. Chaber, Conditions which imply compactness in countably compact spaces, Bull. Acad. Polon. Sci. Sér. Sci. Math. Astronom. Phys. 24 (1976), 993-998.

[Dow88a] A. Dow, Two applications of reflection and forcing to topology, in: General Topology and Its Relations to Modern Analysis and Algebra, VI (Prague, 1986), Res. Exp. Math. 16, Heldermann, Berlin, 1988, 155-172.

[Dow88b] -, An empty class of nonmetric spaces, Proc. Amer. Math. Soc. 104 (1988), 999-1001.

[Dow89] - Compact spaces of countable tightness in the Cohen model, in: Set Theory and Its Applications (Toronto, ON, 1987), Lecture Notes in Math. 1401, Springer, Berlin, 1989, 55-67.

[Gru02] G. Gruenhage, Spaces having a small diagonal, Proc. Internat. Conf. on Topology and its Applications (Yokohama, 1999), Topology Appl. 122 (2002), 183200.

[Huš77] M. Hušek, Topological spaces without $\kappa$-accessible diagonal, Comment. Math. Univ. Carolin. 18 (1977), 777-788.

[JS92] I. Juhász and Z. Szentmiklóssy, Convergent free sequences in compact spaces, Proc. Amer. Math. Soc. 116 (1992), 1153-1160.

[Ku80] K. Kunen, Set Theory: An Introduction to Independence Proofs, North-Holland, Amsterdam, 1980.

[Tod89] S. Todorčević, Partition Problems in Topology, Contemp. Math. 84, Amer. Math. Soc., Providence, RI, 1989. 
[Zho82] H. X. Zhou, On the small diagonals, Topology Appl. 13 (1982), 283-293.

Department of Mathematics

UNC-Charlotte

9201 University City Blvd.

Charlotte, NC 28223-0001, U.S.A.

E-mail: adow@uncc.edu

$$
\text { opavlov@email.uncc.edu }
$$

URL: http://www.math.uncc.edu/ adow

Received 24 November 2005;

in revised form 25 February 2006 